\title{
Taxa de câmbio real e crescimento econômico: uma comparação entre economias emergentes e desenvolvidas
}

\author{
MARCOS ROCHA \\ MARCELO CURADO \\ DANIEL DAMIANI*
}

Real exchange rate and economic growth: a comparison between emerging and developed economies. This paper presents a discussion on the relationship between economic growth and real exchange rate. The article presents the results generated by a dynamic panel that tested the relationship of economic growth with the level of the exchange rate, exchange rate volatility and the choice of exchange rate regime from 26 countries, 13 emerging and 13 developed. The results suggest that the level of the exchange rate and volatility are relevant for growth. Finally, the paper stresses that there are important differences when comparing developed and emerging economies.

Key words: exchange rate; economic growth; economic policy.

JEL Classification: C23; F31; F33.

\section{INTRODUÇÃO}

A ocorrência de superávits no balanço de pagamentos e a utilização da taxa de câmbio como um elemento relevante na estratégia de desenvolvimento econômico de um amplo conjunto de economias emergentes nos últimos anos evidenciaram a importância do câmbio e de suas flutuações para a economia. Neste contex-

\footnotetext{
* Respectivamente: professor da PUC-SP. E-mail: sir.mrocha@gmail.com; professor associado da Universidade Federal do Paraná e bolsista do Programa Cátedras para o Desenvolvimento do IPEA/CAPES. E-mail: curado@ufpr.br; mestre em Desenvolvimento Econômico pela UFPR. E-mail: dani1917@ yahoo.com.br. Submetido: 13/5/2008; aprovado 22/3/2010.
} 
to, o comportamento do câmbio e seus impactos sobre a dinâmica de preços, a capacidade competitiva e a estrutura produtiva dos países voltou a ser tema de amplo debate acadêmico.

A condução da política macroeconômica em distintas economias emergentes confirmou a opção de diversos bancos centrais pela intervenção no mercado de câmbio. Em alguns casos - na China, por exemplo - a utilização do câmbio como componente de uma estratégia de desenvolvimento centrada na ampliação das exportações foi patente ${ }^{1}$. Neste contexto, o processo de ajuste através das livres forças de mercado - em tese o principal mecanismo de ajustamento da taxa no regime de câmbio flutuante - desempenhou um papel marginal.

A literatura econômica tradicional, no entanto, dispensa pouca atenção para o papel da taxa de câmbio e de suas flutuações no processo de crescimento. De forma geral, para esta literatura a política econômica e suas variáveis centrais taxa de juros e taxa de câmbio - não desempenham papel relevante na trajetória de crescimento econômico de um país ${ }^{2}$. Isto reflete a opção metodológica desta literatura que opta por construir modelos macroeconômicos gerais, desconsiderando o caráter específico do processo de crescimento de uma nação ${ }^{3}$.

Em sentido oposto, a literatura econômica de inspiração keynesiana sobre a relevância da taxa de câmbio no processo de crescimento econômico é vasta. Autores como Eichengreen (2004), Edwards (2006) e Bresser-Pereira (2007) têm advogado em favor da importância da taxa de câmbio para o processo de crescimento. Da mesma forma, a discussão sobre o "medo de flutuar", inaugurada por Calvo e Reinhart (2002), deixa evidente a importância do comportamento da taxa de câmbio e de suas flutuações, particularmente em economias emergentes.

De modo geral, tanto apreciações cambiais quanto repentinas desvalorizações são temidas pelos seus efeitos desestabilizadores. As valorizações repentinas são temidas por seus efeitos sobre a competitividade externa da economia, sobretudo em países para os quais o mercado externo é uma fonte importante de demanda agregada. Por outro lado, os processos de forte desvalorização são temidos por seus efeitos sobre a estabilidade dos preços.

As evidências disponíveis, particularmente para os países emergentes, sugerem que a política econômica é um elemento relevante na determinação da trajetória de crescimento. Este trabalho procura contribuir para a discussão, em particular no que se refere à apresentação de evidências empíricas sobre o tema do papel da taxa de câmbio no processo de crescimento. Seu objetivo principal é identificar qual o papel da taxa de câmbio e sua volatilidade no processo de crescimento econômi-

\footnotetext{
${ }^{1}$ Para uma discussão detalhada sobre o papel da taxa de câmbio na estratégia de desenvolvimento chinesa veja-se, entre outros, Eichengreen (2004).

${ }^{2}$ Para uma discussão aprofundada sobre os determinantes do crescimento econômico de acordo com a literatura convencional recomenda-se a leitura de Barro \& Sala-i-Martin (1995).

${ }^{3}$ Para uma discussão sobre o tema de uma perspectiva metodológica, veja-se Bresser-Pereira (2005).
} 
co de um grupo selecionado de países desenvolvidos e emergentes no período posterior à ruptura do acordo de Bretton Woods 4 .

Além dessa introdução, o artigo é dividido da seguinte forma. A segunda seção faz uma breve discussão teórica sobre os efeitos da taxa de câmbio e de suas flutuações no crescimento econômico. A terceira seção apresenta a base de dados e a especificação do modelo econométrico estimado. A quarta seção apresenta as evidências empíricas obtidas e tece alguns comentários. Finalmente, na quinta seção são resumidas as principais conclusões do trabalho.

\section{TAXA DE CÂMBIO E SUA IMPORTÂNCIA NO SISTEMA ECONÔMICO}

Esta seção do trabalho é dedicada ao estudo do papel da taxa de câmbio e de suas flutuações no processo de crescimento econômico. A seção é organizada da seguinte forma: inicialmente, são apresentas evidências empíricas sobre o comportamento da taxa de câmbio e de sua volatilidade. A questão do "medo de flutuar" também é apresentada nesta seção. $\mathrm{Na}$ sequência procede-se uma breve discussão sobre o papel da taxa de câmbio enquanto elemento de política econômica, com ênfase no papel da variável sobre a competitividade externa e a evolução do balanço de pagamentos. Por fim, a última parte desta seção é dedicada ao estudo do papel específico da volatilidade cambial para o crescimento.

\section{Regimes cambiais de jure e de facto: a questão do medo de flutuar}

O período pós Bretton Woods foi marcado tanto por momentos de alta liquidez no mercado financeiro internacional como por crises de confiança com fuga de capitais dos mercados emergentes. Na primeira metade da década de 1990 os países emergentes latino-americanos e do Leste Asiático beneficiaram-se da ampliação da liquidez internacional para realizar uma série de programas de estabilização econômica. Tais programas utilizaram-se extensivamente desta ampla liquidez internacional, num contexto de abertura comercial e da conta de capitais, para valorizar suas moedas e, a partir deste processo de "ancoragem cambial", promover a estabilização dos preços domésticos. O limite desta estratégia ficou evidente a partir da ocorrência sistemática de crises cambiais nessas economias 5 . Após o aban-

\footnotetext{
${ }^{4}$ Esse marco histórico foi escolhido por ter significado uma quebra de padrão em termos de adoção de regimes cambiais; isto é, a partir do fim de Bretton Woods, o regime de câmbio fixo foi gradativamente sendo abandonado pela maioria dos países do mundo. Segundo o FMI, em 1970, 97,2\% dos países-membros tinham taxa de câmbio fixas. Em 1980 esse número já cai para 38,9\% e, em 1999, para $11,1 \%$. Com regimes de câmbio flutuantes, a volatilidade cambial se torna uma variável relevante a ser considerada para o entendimento do ciclo econômico.

${ }^{5}$ México em 1994, Sudeste da Ásia em 1997, Rússia em 1998 e Brasil em 1999 são exemplos dos limites dos programas de estabilização econômica através de "ancoragem cambial” em países emergentes.
} 
dono dos regimes de administração do câmbio, difundiu-se de forma ampla a utilização de regimes de câmbio flutuante com intervenção (dirty floating).

A Tabela 1 ilustra - de uma perspectiva histórica mais ampla — essa elevação do número de países que utilizam o câmbio flutuante de jure. Pode ser percebido que o regime de câmbio flexível, levando em consideração a classificação do Fundo Monetário Internacional (FMI), cresceu de forma praticamente contínua desde o final dos anos 1980. Como exceção a esta regra, vale a pena destacar a primeira metade da década de 1990 em que se verificou uma importante ampliação dos regimes de câmbio administrado.

Tabela 1: Classificação dos regimes cambiais

\begin{tabular}{c|cccc}
\hline \multirow{2}{*}{ Ano } & \multicolumn{4}{|c}{ Porcentagem de países classificados pelo FMl como tendo: } \\
\cline { 2 - 5 } & Fixo & Flexibilidade limitada & Administrada & Flexível \\
\hline 1970 & 97,2 & 0,0 & 0,0 & 2,8 \\
\hline 1975 & 63,9 & 11,1 & 13,9 & 11,1 \\
\hline 1980 & 38,9 & 5,6 & 47,2 & 8,3 \\
\hline 1985 & 33,3 & 5,6 & 36,1 & 25 \\
\hline 1990 & 19,4 & 13,9 & 30,6 & 36,1 \\
\hline 1995 & 13,9 & 8,3 & 38,9 & 38,9 \\
\hline 1999 & 11,1 & 11,1 & 33,3 & 44,5 \\
\hline
\end{tabular}

Fonte: Calvo e Reinhart (2002)

Calvo e Reinhart (2002), no entanto, advogam que esta classificação do FMI não corresponde ao comportamento de facto dos países emergentes. Seu trabalho analisa o comportamento das flutuações da taxa de câmbio para um amplo conjunto de economias que adotam de jure regimes de flutuação cambial. Seus resultados são claros em demonstrar que diversas economias que declararam praticar flutuação cambial administravam a sua taxa de câmbio.

Os autores analisam o comportamento da taxa de câmbio para 39 países durante o período que se estende de janeiro de 1970 a dezembro de 1999. O argumento do medo de flutuar é inicialmente testado da seguinte forma. Escolhe-se como valor crítico para análise uma faixa de variação mensal da taxa de câmbio de $\pm 2,5 \%$. De acordo com os autores, economias que adotassem um regime de câmbio administrado deveriam ter uma probabilidade relativamente elevada de que as flutuações mensais da taxa de câmbio não fossem superiores a faixa estabelecida de $\pm 2,5 \%$ no mês. Por outro lado, economias que adotassem a flutuação cambial deveriam ter uma probabilidade relativamente menor de que a variação da taxa de câmbio ficasse no interior desta faixa

A Tabela 2, extraída do trabalho original de Calvo e Reinhart (2002), apresenta um amostra da discussão. 
Tabela 2: Volatilidade da Taxa de Câmbio para

Economias Selecionadas que adotam regime de flutuação cambial

\begin{tabular}{|c|c|c|}
\hline País & Período & $\begin{array}{l}\text { Probabilidade de que a variação } \\
\text { mensal da taxa de câmbio esteja } \\
\text { dentro da banda de } \pm 2,5 \%\end{array}$ \\
\hline Austrália & Jan 1984 - Nov1999 & 70,3 \\
\hline Bolívia & Set 1985 - Dez 1997 & 93,9 \\
\hline Canadá & Jun 1970 - Nov 1999 & 93,6 \\
\hline Índia & Mar 199 - Nov 1999 & 93,4 \\
\hline Quênia & Out 1993 - Dez 1997 & 72,2 \\
\hline Japão & Fev 1973 - Nov 1999 & 61,2 \\
\hline México & Dez 1994 - Nov 1999 & 63,5 \\
\hline Nova Zelândia & Mar 1985 - Nov 1999 & 72,2 \\
\hline Nigéria & Out 1986 - Mar 1993 & 74,5 \\
\hline Noruega & Dez 1992 - Dez 1994 & 95,8 \\
\hline Peru & Ago 1990 - Nov1999 & 71,4 \\
\hline Filipinas & Jan 1988 - Nov 1999 & 74,5 \\
\hline África do Sul & Jan 1983 - Nov 1999 & 66,2 \\
\hline Espanha & Jan 1984 - Maio 1989 & 93,8 \\
\hline Suécia & Nov 1992 - Nov 1999 & 75,5 \\
\hline Uganda & Jan 1992 - Nov 1999 & 77,9 \\
\hline Estados Unidos /DM & Fev 1973 - Nov 1999 & 58,7 \\
\hline
\end{tabular}

Fonte: Calvo \& Reinhart (2002)

Para a economia norte-americana, por exemplo, a probabilidade de que a taxa de câmbio em relação ao marco alemão flutuasse dentro da faixa preestabelecida de $\pm 2,5 \%$ era de apenas $59 \%$. Por outro lado, para economias como a Bolívia e a Índia, que praticavam no período analisado o câmbio flutuante, a probabilidade de que a flutuação cambial ficasse dentro da faixa preestabelecida era de aproximadamente $95 \%$. Em outras palavras, de acordo com Calvo e Reinhart (2002), a probabilidade de que taxa de câmbio nessas duas economias flutuasse acima da faixa de $\pm 2,5 \%$ ao mês era de apenas $5 \%$.

Os autores também estudam o comportamento de um grupo de países que declaram utilizar o câmbio flutuante com intervenção. Como resultado geral deste grupo vale a pena destacar o seguinte resultado:

"On average, there is an 88 percent probability that managed floaters' monthly changes in the exchange rate are conned to this narrow band. 
This exchange rate stability versus the U.S. dollar (or DM if it is a European country) is surprising in light of the fact that for many emerging market countries during these episodes, in inflation rates were well above U.S. or German levels, terms-of-trade shocks were frequent and large, and macroeconomic fundamentals were markedly more volatile than in any of the benchmark countries." (Calvo e Reinhart, 2002, p. 389)

O medo de flutuar nas economias emergentes poderia ser explicado pela conjunção de dois fatores: problemas de credibilidade e o elevado efeito pass-through que caracterizam estes países. De acordo com Calvo e Reinhart (2002), embora os países emergentes sejam heterogêneos, eles partilham de uma característica comum: a falta de credibilidade, particularmente da autoridade monetária.

Calvo e Reinhart (2002) distinguem ainda outras razões para o medo de flutuar que não se encontram associadas diretamente associados ao tema da credibilidade. Por exemplo, as desvalorizações em países emergentes (ou fortes depreciações) tendem a ser associadas a severas recessões. A ocorrência de defaults e as dificuldades relacionadas ao gerenciamento dos serviços da dívida dos países podem ser aumentadas num contexto de maior flexibilidade, onde é permitido que a taxa de câmbio flutue de forma significante.

Finalmente, os autores sugerem que o pass-through dos países emergentes é maior do que o verificado nos países desenvolvidos. Esta observação explica em especial para aqueles países que adotam regimes de metas de inflação, onde a preocupação dos policy-makers concernente aos movimentos de preços é particularmente alta - a tendência a reduzir as flutuações da taxa de câmbio, em especial os movimentos de desvalorização da moeda tendo vista seus impactos inflacionários.

Ainda de acordo com os autores, os países emergentes possuem um viés no sentido de aumentar a volatilidade da taxa de juros. Isso significa que, para esses países, em face da escolha entre estabilizar a taxa de juros ou estabilizar a taxa de câmbio, a preferência em geral é por estabilizar a taxa de câmbio. Segundo estas análises, os governos consideram satisfatória alguma volatilidade nas duas variáveis, preferindo, entretanto, reduzir a variação da taxa de câmbio.

Ho e McCauley (2002) confirmam os resultados esperados por Calvo e Reinhart (2002). A conclusão central de seu trabalho é que nos países emergentes o efeito pass-through é superior ao verificado para os países desenvolvidos. De acordo com os autores, este resultado reflete o papel da taxa de câmbio na formação das expectativas sobre inflação nas economias emergentes. Nessas economias, o histórico de alta inflação e de crises no balanço de pagamentos torna as expectativas dos agentes sobre o comportamento da inflação mais sensível às desvalorizações cambiais.

Em suma, considerando que a maioria dos recém-convertidos à flutuação pratica na verdade formas "disfarçadas" de administração da taxa de câmbio, a literatura recente conclui que a ideia usual de que os chamados regimes cambiais intermediários estão desaparecendo é um mito. A preocupação com a taxa de câmbio 
e suas flutuações reforça o argumento favorável à importância da variável para a economia. A próxima seção é dedicada à realização de uma breve discussão sobre o tema.

\section{Taxa de câmbio, competitividade externa e estratégia de desenvolvimento}

A literatura de inspiração keynesiana sobre a relevância da taxa de câmbio no sistema econômico é vasta. A preocupação com o papel desempenhado pelo câmbio na economia é particularmente acentuada quando se analisa o comportamento das economias emergentes. Edwards (2006, p. 28) afirma que: "The exchange rate is one of most important macroeconomic variables in the emerging and transition countries. It affects inflation, exports, imports and economic activity". De forma geral, a taxa de câmbio e seus impactos sobre a competitividade externa dos países são analisados como elementos integrantes de uma estratégia de desenvolvimento econômico.

A experiência histórica é farta em exemplos de como a taxa de câmbio foi utilizada como instrumento de uma estratégia de desenvolvimento. Eichengreen (2004), por exemplo, destaca a importância recente da administração das taxas de câmbio nos países emergentes da Ásia como elemento de sua estratégia de promoção do crescimento econômico.

"[...] pegged exchange rates and resistance to pressures for revaluation as their economies and current accounts strengthen have been at the center of their development strategies [...] There is no question that their accumulation of reserves is a concomitant of intervention in the foreign exchange market to keep their currencies down, which is in turn a concomitant of the strategy of promoting exports as a way of stimulating growth.” (Eichengreen, 2004, p. 2)

Dessa forma, ao não permitir que a maior liquidez internacional e os superávits no balanço de pagamentos se traduzissem em fortes valorizações de suas moedas, os países de Ásia asseguraram a manutenção de sua competitividade externa, o que se constituiu num elemento central de sua estratégia de desenvolvimento econômico.

A importância da taxa de câmbio no sistema econômico é igualmente sublinhada por Bresser-Pereira (2007). Segundo o autor:

"A armadilha da taxa de câmbio é mais letal que a da taxa de juros, porque ela facilmente termina em crise de balanço de pagamentos, enquanto a primeira pode terminar em crise de dominância fiscal, embora seja difícil que isso ocorra. A taxa de câmbio apreciada reduz exportações e aumenta importações. Os índices de endividamento externo do país se deterioram, as perspectivas de piora na margem se agravam e, subitamente, os credores decidem suspender a rolagem da dívida externa, como aconteceu em 1998 e 2002.”(Bresser-Pereira, 2007, p. 244). 
A valorização cambial é, portanto, associada à piora nas contas externas, que em última análise se traduz numa crise no balanço de pagamentos, em decorrência da piora dos indicadores externos de endividamento. Há, portanto, uma "armadilha" no processo de crescimento associada à valorização cambial e à deterioração das condições de competitividade externa.

A importância da competitividade externa e a manutenção de uma taxa de câmbio competitiva no processo de desenvolvimento econômico são fatores igualmente destacados por Netto (2005). Segundo o autor:

"Existe uma hierarquia entre os problemas que o desenvolvimento enfrenta. No topo encontra-se o obstáculo externo: a negligência com as exportações, a persistência por longos períodos de saldos negativos em transações correntes e a decorrente elevação da dívida levam o país à dependência externa e à estagnação. Foram esses os fatores que deram origem às três crises $(1963,1981$ e 1999) [...] Uma situação externa confortável não produz automaticamente o crescimento, mas o inverso produz estagnação. Para o crescimento autossustentado, é fundamental uma taxa de câmbio competitiva." (Netto, 2005, p. 251)

O efeito da valorização cambial sobre a estrutura produtiva da economia é outro elemento discutido por essa literatura. A conjuntura econômica recente que combina ampla liquidez no mercado financeiro internacional e elevação dos preços das commodities exportadas por alguns países emergentes - tornou mais intensa a discussão sobre os efeitos de longo prazo da taxa de câmbio valorizada.

Os superávits obtidos pelos países emergentes nesse contexto contribuiriam para valorizar o câmbio, reduzindo a competitividade da economia, particularmente de setores exportadores de produtos industrializados com maior valor agregado e/ou conteúdo tecnológico. A valorização cambial contribuiria, portanto, para reduzir a rentabilidade de uma série de atividades com maior valor agregado/conteúdo tecnológico, gerando desta forma uma tendência de aumento da participação dos setores exportadores de commodities na economia. Este é, em síntese, o resultado central literatura que estuda a "doença holandesa".

Esse aumento é preocupante por diversas razões, com destaque para a menor elasticidade-renda e maior elasticidade preço de demanda que caracterizam no longo prazo os setores exportadores de commodities.

Em síntese, a valorização cambial contribuiria de forma decisiva para alterar a estrutura produtiva da economia, elevando a participação dos setores exportadores de commodities com menor elasticidade-renda e maior elasticidade preço da

\footnotetext{
${ }^{6}$ Para uma discussão mais desenvolvida sobre o tema recomenda-se a leitura de Bresser-Pereira (2007) e Palma (2005).
} 
demanda, o que, desde que seja válida a lei de Thirlwall 7 , implicaria uma menor taxa de crescimento com equilíbrio no balanço de pagamentos no longo prazo.

A ocorrência dessa elevação da participação de commodities no total exportado é ainda mais preocupante para os países emergentes exportadores de petróleo e gás. A preocupação com efeitos do câmbio sobre a estrutura produtiva da economia ultrapassa as fronteiras da literatura de inspiração keynesiana. Greenspan (2007), por exemplo, alerta para o problema no caso específico da Rússia:

"Os sintomas da doença holandesa já são evidentes. Com o aumento das exportações de petróleo e de gás, o valor do rublo disparou e o valor das demais exportações russas ficou para trás. Entre 1998 e 2006, o valor do rublo em relação às moedas dos parceiros comerciais da Rússia dobrou, depois do ajuste pelas respectivas taxas de inflação. O impacto era previsível: as demais exportações, com exclusão das de petróleo e de gás, aumentaram em termos reais a taxas correspondentes à metade das de expansão das exportações de petróleo e gás.” (Greenspan, 2007, p. 317)

A relação entre a taxa de câmbio e competitividade externa, especialmente em economias emergentes, se constitui, portanto, num elemento relevante da discussão de inspiração keynesiana sobre o papel da taxa de câmbio na economia. As evidências recentes, particularmente a utilização de uma estratégia de crescimento puxado pelas exportações na qual os bancos centrais procuram conter valorizações cambiais excessivas, reforçam os argumentos da literatura keynesiana em favor da importância da taxa de câmbio na economia.

\section{Volatilidade cambial e crescimento econômico}

Esta seção é dedicada exclusivamente ao estudo do papel da volatilidade cambial no processo de crescimento econômico. Até o momento, a literatura identificou alguns canais de transmissão dos efeitos da volatilidade da taxa real de câmbio no processo de crescimento econômico, dentre os quais merecem destaque: i) o papel do desenvolvimento do mercado financeiro; ii) o grau de abertura comercial; iii) as decisões de investimento sob incerteza e iv) o histórico de alta inflação na experiência internacional de alguns países.

A relação entre volatilidade da taxa de câmbio e o crescimento econômico tem recebido mais atenção dos economistas teóricos desde o começo da década de 1990. Trabalhos empíricos sobre o tema são menos frequentes, mas não inexistentes.

\footnotetext{
${ }^{7}$ Em sua versão original a lei afirma que a taxa de crescimento com equilíbrio do balanço de pagamentos é igual à taxa de crescimento da economia mundial ponderada pela relação entre as elasticidades-renda de exportações e importações. Dessa forma, para uma mesma taxa de crescimento mundial, quanto menor a elasticidade-renda das exportações em relação à elasticidade-renda das importações, menor a taxa de crescimento com equilíbrio do balanço de pagamentos para a economia. A discussão original da lei é apresentada em Thirlwall (1979).
} 
Ramey e Ramey (1995), por exemplo, encontraram uma correlação negativa e significante entre volatilidade da taxa de câmbio real e crescimento econômico.

Aghion et al (2006) testa a hipótese de que países com sistemas financeiros menos desenvolvidos são mais afetados pela volatilidade cambial. Os autores apresentam um modelo de uma economia pequena com rigidez de salários na qual os choques exógenos são causados pela volatilidade cambial. O crescimento é medido pelo aumento na produtividade via investimento. O desenvolvimento do sistema financeiro - medido pela proporção do crédito em relação ao PIB — ganha importância quando os proprietários das firmas têm duas opções diante do choque cambial: endividam-se e continuam investindo, ou se protegem do choque cessando os investimentos. Fica claro nesse ponto que, em países que têm um sistema de crédito desenvolvido, o prêmio ao risco é muito mais acessível.

Os autores testaram essa hipótese para um conjunto de 83 países usando um painel dinâmico e encontraram resultados que, em sua grande maioria, corroboram a hipótese proposta. Em países menos desenvolvidos, que geralmente possuem um mercado financeiro mais incipiente do que o dos países desenvolvidos, quanto mais flexível for a taxa de câmbio, mais a volatilidade da taxa de câmbio real afeta o crescimento. Esse resultado é importante porque, por um lado, desmistifica a ideia de que uma taxa de câmbio flutuante ajuda a estabilizar o produto via comércio externo e, por outro, evidencia a ideia de que alguns modelos, que explicam satisfatoriamente o comportamento de economias desenvolvidas, não têm o mesmo apelo empírico quando se trata das economias emergentes.

O efeito da volatilidade cambial sobre as decisões de investimento é outro canal de transmissão estudado pela literatura econômica em suas vertentes keynesiana e convencional.

Davidson (2002), por exemplo, explora o tema de uma perspectiva pós-keynesiana, ou seja, na qual a incerteza é um elemento recorrente da tomada de decisões, particularmente na irreversível decisão de investimento. O autor propôs que, em uma economia aberta, uma taxa de câmbio volátil aumenta o grau de incerteza com relação ao futuro; essa incerteza, por sua vez, aumenta a preferência pela liquidez e, portanto, eleva a taxa de juros. Por outro lado, a ampliação da incerteza sobre o comportamento futuro da economia tende a diminuir a eficiência marginal do capital. Tais efeitos contribuem, em seu conjunto, para a redução dos gastos com investimento e, portanto, do nível de demanda agregada do sistema.

Segundo Jones, Manuelli e Stacchetti (2000), o que determina qual efeito será maior é a elasticidade intertemporal de substituição entre taxa de juros e de câmbio: apenas na situação em que a mesma seja maior que 1, a volatilidade terá um efeito negativo no investimento e, consequentemente, no crescimento. Os resultados encontrados por Angeletos (2003) mostram que em economias cuja capacidade de lucro por investimento não é exaurida, o efeito da volatilidade do câmbio no investimento continua negativo.

Aghion et al (2006) argumentam que não é a queda no investimento que causará a maior consequência negativa no crescimento, mas sim a "qualificação" desse investimento. A incerteza causada por uma alta volatilidade, segundo os autores, 
faz com que os agentes substituam investimentos de longo prazo por investimentos de curto prazo. Nesse contexto, o problema é que entre os investimentos de longo prazo estão os investimentos em $\mathrm{P} \& \mathrm{D}$, que representam o investimento responsável pelo crescimento de longo prazo em qualquer economia.

Sérven (2002) testa e confirma a hipótese de que nos países em desenvolvimento as consequências negativas de uma alta taxa de volatilidade no câmbio sobre decisões de investimento são mais acentuadas que nos países desenvolvidos. $\mathrm{O}$ autor argumenta que nos países em desenvolvimento as incertezas são maiores. Isso acontece em face dos choques de credibilidade no passado, típicos das economias emergentes, e seu baixo desenvolvimento do sistema financeiro. Incerteza maior significa, segundo o autor, uma taxa menor de investimento.

O grau de abertura do comércio é outro canal de transmissão da volatilidade cambial para o crescimento. Segue-se aqui a tradição de modelos como o de Mundell-Fleming (1962), em que é postulado que o setor externo é uma fonte de demanda agregada e, consequentemente, influencia o crescimento econômico ${ }^{8}$.

O grau de abertura comercial é uma discussão que nos leva invariavelmente a discutir a relação entre volatilidade cambial e comércio internacional. $\mathrm{Na}$ literatura encontramos um problema de dupla causalidade entre essas duas variáveis: o aumento do comércio tem impacto sobre a volatilidade cambial, e a volatilidade cambial impacta o comércio. Alguns trabalhos tiveram esse problema de endogeneidade, pois apenas captaram, por exemplo, a influência do comércio sobre a volatilidade. Esse é o caso do trabalho de Tenreyro e Barro (2002) e de Tenreyro (2003).

Broda e Romalis (2003), ao contrário, elaboraram um modelo que capta os dois movimentos. Primeiramente, testaram e comprovaram a hipótese de Mundell (1961) de que o fluxo de comércio internacional ajuda a estabilizar flutuações da taxa de câmbio real e, portanto, diminui a volatilidade cambial. Na sequência testaram a hipótese de que a volatilidade cambial influencia negativamente o comércio internacional. Sobre esse segundo teste os autores encontraram resultados interessantes: o primeiro é que, de fato, a volatilidade cambial afeta negativamente as exportações; o segundo é que esse efeito negativo é muito pequeno para produtos agrícolas e é razoavelmente grande para produtos industrializados; e, por fim, que quanto maior for a distância entre um país e o seu principal parceiro comercial maior será a sua volatilidade cambial, resultado que evidencia o incentivo à formação de blocos regionais.

Finalmente, vale retomar a discussão do efeito da volatilidade cambial sobre a inflação. Segundo Ho e McCauley (2002), esse efeito é maior em países emergentes, em virtude do histórico de inflação alta que muitos deles apresentaram ao longo da história. Com alta volatilidade cambial, a pressão inflacionária viria dos

\footnotetext{
${ }^{8}$ Não cabe aqui uma discussão histórica sobre o crescimento via demanda agregada $v s$ oferta agregada. O importante é ser definido que, como consenso da maioria dos macroeconomistas, a demanda agregada, no que tange à teoria na qual esse trabalho está embasado, tem a capacidade de impulsionar o crescimento do produto, ao menos no curto prazo.
} 
reajustes dos produtos importados. Nesse cenário, as autoridades monetárias seriam obrigadas a aumentar as taxas de juros para conter a inflação, comprometendo, entretanto, a performance de crescimento ao desestimular as decisões de investimento.

\section{BASE DE DADOS E O MODELO ECONOMÉTRICO}

Os dados do painel de crescimento foram coletados World Penn Tables 6.2, construída por Heston, Summers e Atina em 2006 e do World Development Indicators 2008 do Banco Mundial (como detalhado a seguir). A amostra utilizada é um painel com séries temporais que cobrem os anos de 1971 a 2006.

\section{Classificação dos regimes cambiais de facto}

Além disso, é utilizada a classificação de regimes cambiais construída por Reinhart e Rogoff (2002) para as dummies de regimes de câmbio. Essa nova classificação deu novo fôlego a essa agenda de pesquisa; na década de 90 alguns autores, tais como Ghosh et al. (1997) e o próprio FMI (1997), não encontraram qualquer relação entre a escolha do regime cambial e o crescimento econômico. Calvo e Reinhart (2002) e Levy, Yeyati e Sturzenegger (1999) foram os primeiros a criticar esses trabalhos, atribuindo essa falta de relação ao uso da classificação oficial do fundo dos regimes cambiais, no caso os regimes de júri. Reinhart e Rogoff (2002) então desenvolveram uma reclassificação histórica dos regimes de câmbio, tanto na taxonomia quanto nos valores das séries de taxas de câmbio. Para construir a classificação foi empregada uma base de dados extensiva das taxas de câmbio determinadas no "mercado paralelo". O algoritmo de classificação mais acurado dos autores levou a uma reestruturação ampla da classificação dos regimes para diversos países e períodos.

Os autores chamam a atenção para o fato de que, na maior parte das vezes em que a categorização oficial aponta alguma forma de câmbio fixo, sua nova metodologia revela que o verdadeiro regime monetário em voga é algo radicalmente diferente; muitas vezes, uma variante de flutuação. De forma análoga, o trabalho revela que quando a classificação anunciada é de regime flutuante, rotineiramente a metodologia mostra que, na realidade, trata-se de uma forma de regime de certa rigidez de facto.

Muitos estudos utilizam uma classificação-padrão de regimes cambiais, como o Annual Report on Exchange Rate Arrangements and Exchange Restrictions, publicadas pelo Fundo Monetário Internacional (FMI) ${ }^{9}$. Entretanto, uma leitura

\footnotetext{
${ }^{9}$ O Fundo, reconhecendo as limitações de sua classificação inicial, revisou e renovou a classificação oficial a partir de 1997, embora não tenha reavaliado sua classificação histórica depois disso. Um problema com a classificação pré-1997 que tem recebido substancial atenção na literatura recente é a frequência de episódios em que o regime é classificado como flutuante (gerenciado ou não) quando, de fato, o país tinha um regime de câmbio de facto fixo ou crawling peg.
} 
mais acurada das experiências de regimes cambiais sugere que essas classificações oficiais falham muitas vezes em descrever as práticas dos países, e que o gap entre de facto e de jure ${ }^{10}$ pode ser extenso. Poucos estudos prévios tentaram estender a classificação oficial do FMI de quatro padrões; alguns se basearam apenas em métodos puramente estatísticos para reclassificar os grupos de práticas cambiais.

A taxonomia dos arranjos de regimes cambiais inclui 14 classificações e é apresentado no Quadro 1. O menos flexível dos arranjos é assinalado com os menores valores na escala, seguindo o Quadro 1 - entretanto, no caso da classificação ampla, é necessário tratar "freely falling" 11 como uma categoria separada, como sugerem os autores da classificação. Por simplicidade, e seguindo Coudert e Couharde (2008), este artigo utiliza as três categorias amplas em suas estimações, e denota os regimes da seguinte forma: Fixo, Intermediário e Flexível.

Quadro 1: Categorização detalhada e categorização ampla dos regimes cambiais

\begin{tabular}{|c|c|c|}
\hline Categoria de regime cambial & $\begin{array}{l}\text { Número associado } \\
\text { à categoria }\end{array}$ & $\begin{array}{l}\text { Classificação } \\
\text { cambial utilizada } \\
\text { neste trabalho }\end{array}$ \\
\hline Sem separação legal & 1 & Fixo \\
\hline Peg anunciado ou currency board & 2 & Fixo \\
\hline $\begin{array}{l}\text { Banda horizontal pré-anunciada que seja mais } \\
\text { estreita ou igual } a+/-2 \%\end{array}$ & 3 & Fixo \\
\hline Peg de facto & 4 & Fixo \\
\hline Crawling peg pré-anunciado & 5 & Intermediário \\
\hline $\begin{array}{l}\text { Crawling peg pré-anunciado que seja mais } \\
\text { estreita ou igual } a+/-2 \%\end{array}$ & 6 & Intermediário \\
\hline Crawling peg de facto & 7 & Intermediário \\
\hline $\begin{array}{l}\text { Crawling band de facto que seja mais estreita } \\
\text { ou igual } a+/-2 \%\end{array}$ & 8 & Intermediário \\
\hline $\begin{array}{l}\text { Crawling band pré-anunciada que seja mais } \\
\text { ampla ou igual } a+/-2 \%\end{array}$ & 9 & Intermediário \\
\hline $\begin{array}{l}\text { Crawling band de facto que seja mais estreita } \\
\text { ou igual } a+/-5 \%\end{array}$ & 10 & Intermediário \\
\hline
\end{tabular}

continua na pág. 541

\footnotetext{
${ }^{10}$ Uma boa discussão do funcionamento dos regimes cambiais e seus impactos sobre política monetária e da distinção de jure e de facto pode ser vista em Holland e Canuto (2000).

${ }^{11}$ Freely falling é uma nova categoria cunhada por Reinhart e Rogoff (2002) em que estão agrupados essencialmente dois casos especiais: 1 . Economias que apresentaram taxa de inflação anual superior a $40 \%$ e 2. Países que atravessaram crises cambiais. Neste último caso, a configuração de Freely Falling estende-se por seis meses após a ocorrência da crise cambial. Em síntese, a categoria foi criada porque esses desarranjos macroeconômicos caracterizam arranjos cambiais especiais, que devem ser distinguidos da análise de regimes feita unicamente dentro de um contexto de discussão flexibilidade/rigidez. Para maiores detalhes, consultar o trabalho dos autores.
} 


\begin{tabular}{|l|c|c|}
\hline $\begin{array}{c}\text { Banda móvel que seja mais estreita ou igual à } \\
+/-2 \% \text { (permitindo apreciação e depreciação) }\end{array}$ & 11 & Intermediário \\
\hline Flutuação administrada & 12 & Intermediário \\
\hline Flutuação livre & 13 & Flutuante \\
\hline Freely falling & 14 & Flutuante \\
\hline
\end{tabular}

Fonte: Reinhart e Rogoff, 2002.

\section{O cálculo do índice de volatilidade do câmbio real}

O método escolhido para medir a volatilidade da taxa de câmbio foi o de Perre e Steinherr (1989). Uma das principais consequências de uma taxa de câmbio muito volátil é o aumento gradual de incerteza em uma economia; essa incerteza, segundo os autores, afeta, entre outras coisas, a própria volatilidade cambial do futuro. Isto é, períodos em que a taxa de câmbio de uma economia apresenta grande volatilidade tendem a gerar mais volatilidade no futuro. Bittencourt et al. (2007) definiu a medida Perre e Steinherr (PS) da seguinte forma: "[...] é definida pelas experiências passadas a respeito dos valores máximo e mínimo da taxa de câmbio, as quais são ajustadas pela experiência do ano anterior relativa a uma taxa de câmbio de equilíbrio". A equação da medida de volatilidade PS é a seguinte:

$$
V_{i t}=u_{i t}=\frac{\max X_{i t-k}^{t}-\min X_{i t-k}^{t}}{\min X_{i k-t}^{t}}+\left[1+\frac{X_{i t}-X_{i t}^{k}}{X_{i t}^{k}}\right]
$$

onde: $k$ é a duração do período; $\min X_{i t}^{k}$ é o valor absoluto mínimo da taxa de câmbio real nos últimos $k$ períodos; $\max X_{i t}^{k}$ é o valor absoluto máximo da taxa de câmbio real dos últimos $k$ períodos; $X_{i t}^{k}$ é a média dos valores absolutos da taxa de câmbio real para os últimos $k$ períodos ${ }^{12}$. A variável $k$ representa o período de um ano.

\section{Depreciação relativa do câmbio}

Além da volatilidade do câmbio, é testada a possibilidade de efeitos da variável sobre o crescimento quando desalinhada com relação à trajetória de longo prazo - mais especificamente, quando se encontra relativamente desvalorizada. Para o modelo econométrico utilizado, foi construído um índice de depreciação cambial relativa, que é a diferença entre a taxa de câmbio real contemporânea $\theta_{i t}$, e a taxa de câmbio real de longo prazo, que equilibra intertemporalmente o balanço de pagamentos. Esta taxa de câmbio real de longo prazo, $\hat{\theta}_{i t}$, foi extraída da série contemporânea $\theta_{i t}$ com filtro Hodrick Prescott. ${ }^{13}$

\footnotetext{
${ }^{12}$ Ese valor é uma proxy da taxa de câmbio de equilíbrio. Para este trabalho calculamos esse valor usando uma média aritmética simples da taxa de câmbio real defasada em dois períodos.

${ }^{13}$ A taxa real de câmbio utilizada é bilateral com relação ao dólar americano, obtida das séries XRAT/ PPP das séries da World Penn Tables 6.2.
} 
$\operatorname{In}(\text { DepCambial })_{i t}=\ln \theta_{i t}-\ln \hat{\theta}_{i t}$

O modelo empírico especificado para este trabalho, portanto, é o seguinte ${ }^{14}$ : $\ln y_{i t}=\beta_{1} \ln y_{i t-1}+\alpha_{1}(I N V / G D P)_{i t}+\alpha_{2} \Delta P O P_{i t}+\alpha_{3} R 1+\alpha_{4} R 2+\alpha_{5} R 3+\alpha_{6} P S_{i}+\ln (\text { DEPCambial })_{i t}+e_{i t}$

onde:

1n $y_{i t}$ representa o log do produto per capita do país $i$;

1n $y_{i t-1}$ representa a variação do produto per capita do país $i$ defasada em um período;

$(I N V / G D P)$ representa a variação do investimento como parcela do produto do país $i$ - séries da World Penn Tables 6.2;

$\triangle P O P_{i t}$ representa o crescimento da população do país $i$ - séries do World Development Indicators do Banco Mundial;

$P S_{i t}$ representa a medida de volatilidade cambial;

R1, R2 e R3 são os regimes cambiais de facto dos países, representando, respectivamente: Fixo; Intermediário; Flutuante;

PS representa a medida de volatilidade da taxa de câmbio do país $i$ no período $t$, como detalhado anteriormente;

$1 n(\text { DepCambio })_{i t}$ é o índice de competitividade externa, gerada com as séries XRAT, PPP, e o filtro Hodrick-Prescott da série de câmbio real. As séries vêm da World Penn Tables 6.2, cuja construção foi detalhada anteriormente.

$e_{i t}$ representa o termo resíduo estocástico normal.

\section{RESULTADOS EMPÍRICOS}

Para a estimação da função de crescimento, foi utilizada a técnica de painel dinâmico, como é comum na literatura da área. A estrutura de análise de painel para o estudo do crescimento do produto tem seus méritos particulares em contraposição às regressões cross-country convencionais, onde só se pode mensurar ou determinar aqueles fatores que são tangíveis o suficiente para se instituir uma medida de indicadores que sejam internacionalmente comparáveis. Entretanto, como sublinhado em Nelson (1998), fatores intangíveis, tais como a organização e as instituições - dimensões da interação social que não podem ser diretamente observados ou medidos - também têm impactos no caminho do crescimento da economia. Como leva em conta os efeitos individuais de um país em consideração, o instrumental do painel econométrico permite o controle da heterogeneidade desses fatores específicos dos países que não são observáveis, e os quais se podem

\footnotetext{
${ }^{14}$ Cabe notar que a inserção de variáveis que descrevem os regimes cambias tem o interesse não só de mostrar o impacto dos regimes sobre o crescimento, mas serve como variável de controle para estudar a volatilidade, permitindo a agregação de países e períodos no tempo em que o câmbio foi controlado ou deixado flutuar livremente.
} 
razoavelmente assumir permanecerem constantes dentro do período em investigação (Peneder, 2004). Por isso, sua adequação para estudar crescimento.

Há uma literatura bastante ampla a respeito da estimação de modelos de crescimento supply-side do produto. Embora não exista um único modelo ao qual todos os analistas subscrevam como o ideal, existe um amplo consenso sobre qual seria o "esqueleto" de um modelo de crescimento: seguindo os trabalhos de Levine e Renelt (1992) e Sala-i-Martin (1997), há convergência sobre quais são as variáveis a serem incluídas nos modelos empíricos de crescimento. Muitas das variáveis convencionais são: a taxa de investimento como proporção do PIB, o crescimento populacional, o capital humano etc. Neste trabalho são incluídas como variáveis básicas de crescimento do produto as taxas de crescimento demográfico e as taxas percentuais de investimento bruto em proporção ao PIB. As demais são supostas constantes e específicas aos países.

O estimador utilizado no painel dinâmico é o de métodos generalizados de momentos (Generalized Method of Moments, GMM). Esses estimadores lidam com efeitos temporais não observáveis por meio da inclusão de interceptos específicos ao período. Tratar destes efeitos não é simples. Por isso, o modelo é dinâmico e pode conter regressores endógenos, que são controlados pela instrumentação das suas diferenças e defasagens. Isso é fundamental para contornar o problema de endogeneidade do câmbio e produto no exercício empírico a seguir.

Os instrumentos correspondentes às condições de momentos são valores defasados tanto em nível como em diferença das variáveis explicativas e dependente. Uma vez que, tipicamente, as condições de momento sobreidentificam a regressão do modelo, o método do painel dinâmico permite teste de especificações através do teste de Hansen. Trabalhando sobre os estimadores de Arellano e Bover (1995), Blundell e Bond (1998) desenvolveram um estimador em sistema (System-GMM), que usa condições de momentos adicionais. Os estimadores de Arellano-Bond (1991) e Blundell-Bover (1998) foram considerados adequados para a análise deste trabalho, por permitirem uma especificação dinâmica (permitindo a variável dependente defasada do PIB), e por instrumentalizarem adequadamente variáveis potencialmente endógenas. Para uma descrição detalhada destas metodologias econométricas, ver Baltagi (2005).

Como essa é uma análise comparativa, foram feitas três regressões: uma apenas com os países emergentes e outra com apenas os países desenvolvidos e uma agregando toda a amostra ${ }^{15}$. As Tabelas 3, 4 e 5 mostram os resultados obtidos, respectivamente. Os resultados dos painéis dinâmicos são estatisticamente robustos para todos os experimentos: os testes Hansen e Hansen-in-Difference não rejeitam

\footnotetext{
${ }^{15}$ Foi utilizada a classificação do Fundo Monetário Internacional (FMI), usualmente trabalhada no World Economic Outlook. O bloco dos emergentes é formado por: Brasil, Argentina, Uruguai, Chile, Venezuela, Colômbia, México, Cingapura, Malásia, China, Índia, Coreia do Sul e Tailândia. O bloco dos países desenvolvidos é formado por: G7 (sem Estados Unidos) mais Espanha, Áustria, Suécia, Dinamarca, Austrália, Nova Zelândia e Bélgica.
} 
a validade dos instrumentos, e o teste Arellano-Bond de autocorrelação dos resíduos na segunda defasagem da variável independente é rejeitado. São três especificações: em cada uma delas, uma variável categórica de regime cambial (Fixo, Flexível e Intermediário) é utilizada somente, deixando as outras como categorias base da dummy.

A Tabela 3 mostra os resultados obtidos para a amostra total, contendo países ricos e emergentes. Como de praxe, o PIB per capita defasado em um período é significante, indicando a persistência da série e a motivação do uso do painel dinâmico. Crescimento demográfico não aparece significante (talvez pela frequência anual dos dados, movimentos de muito longo prazo como o dessa variável não sejam relevantes para explicar crescimento). A variável de investimento como proporção do PIB aparece positiva e significante na especificação (iii).

Com relação ao impacto do câmbio real sobre o crescimento, o experimento na Tabela 3 mostra que ele pode se dar tanto através do seu comportamento volátil como através de sua depreciação relativa com relação ao equilíbrio de longo prazo. A variável de volatilidade cambial resulta negativa e estatisticamente significante para as três especificações da Tabela 3. Já o índice de depreciação cambial é positivo e estatisticamente significante para as três especificações. Assim, para a amostra total, o câmbio depreciado atua positivamente para o crescimento do produto, enquanto a volatilidade do câmbio atua negativamente.

Com relação aos regimes cambiais, as especificações mostram resultados significantes para regime fixo (positivo) e para o regime flexível (negativo). Assim, ceteris paribus, pode-se depreender do resultado empírico que regime fixo está associado positivamente ao crescimento dos países da amostra, enquanto o regime flexível atua de forma negativa - embora as magnitudes dos coeficientes sejam pequenas. A variável representando regimes intermediários não resulta significante.

Tabela 3: Resultados do Painel Dinâmico de Crescimento para a amostra total

\begin{tabular}{l|c|c|c}
\hline & (i) & (ii) & \multicolumn{1}{c}{ (iii) } \\
\hline In(PIB per capita_1) & 0,912 & 0,945 & 0,924 \\
Crescimento Demográfico & $(0,051)^{*}$ & $(0,045)^{*}$ & $(0,051)$ \\
& $9,87 \mathrm{E}-07$ & $5,08 \mathrm{E}-07$ & $8,66 \mathrm{E}-07$ \\
In(Investimento/GDP) & $(8,10 \mathrm{E}-07)$ & $(7,29 \mathrm{E}-07)$ & $(7,98 \mathrm{E}-07)$ \\
Volatilidade Cambial & 0,033 & 0,028 & 0,034 \\
& $(0,021)$ & $(0,021)$ & $(0,020)^{*}$ \\
In(Depreciação Cambial) & $-0,034$ & $-0,040$ & $-0,043$ \\
& $(0,008)^{*}$ & $(0,007)^{*}$ & $(0,007)^{*}$ \\
Regime Fixo & 0,058 & 0,044 & 0,041 \\
& $(0,017)^{*}$ & $(0,018)^{*}$ & $(0,016)^{*}$ \\
Regime Flexível & 0,006 & $\ldots$ & \\
& $(0,003)^{*}$ & $\ldots$ & \\
\hline
\end{tabular}




\begin{tabular}{l|c|c|c}
\hline Regime Intermediário & $\ldots$ & $\ldots$ & 0,012 \\
Intercepto & $\ldots$ & $\ldots$ & $(0,011)$ \\
& 0,649 & 0,433 & 0,564 \\
& $(0,333)^{*}$ & $(0,285)$ & $(0,357)^{*}$ \\
\hline Teste AR(1) & 0,004 & 0,003 & 0,004 \\
Teste AR(2) & 0,087 & 0,087 & 0,103 \\
Teste Hansen & 0,644 & 0,55 & 0,15 \\
Teste Hansen-in-difference & 0,588 & 0,492 & 0,609 \\
\hline N. de observações & 773 & 773 & 773 \\
\hline
\end{tabular}

Fonte: Resultados da pesquisa.Os números entre parênteses são os desvios-padrão; e * indica pelo menos $5 \%$ de nível de significância.

OBS.: As variáveis de regimes cambiais e volatilidade cambial são tratadas como endógenas pelos instrumentos defasados e diferenciados no System-GMM.

Como o objetivo deste trabalho é comparar as economias emergentes com as desenvolvidas, foram realizadas mais duas regressões: a Tabela 4 mostra os resultados dos países emergentes e a Tabela 5 mostra o resultado para os países desenvolvidos.

O resultado muda quando tratamos a amostra apenas para os países emergentes. A variável de depreciação cambial permanece positiva e significante para o crescimento dos países da amostra, mas a magnitude do coeficiente é muito maior, indicando que a importância do câmbio para o crescimento é bem maior para os países emergentes. A variável de volatilidade cambial tem resultados idênticos ao do experimento anterior, atuando negativamente sobre o crescimento. Com relação aos regimes cambiais, o regime fixo continua sendo a variável significante e positiva. Regimes de câmbio flexíveis e intermediários, contudo, resultam sem significância estatística ${ }^{16}$.

Tabela 4: Resultados do Painel Dinâmico de Crescimento para Países Emergentes

\begin{tabular}{l|c|c|c}
\hline & (i) & (ii) & \multicolumn{1}{c}{ (iii) } \\
\hline PIB per capita_1 & 0,967 & 0,966 & 0,960 \\
& $(0,010)^{*}$ & $(0,011)^{*}$ & $(0,009)^{*}$ \\
Crescimento Demográfico & $1,58 \mathrm{E}-08$ & $7,6 \mathrm{E}-09$ & $-9,10 \mathrm{E}-09$ \\
& $(5,96 \mathrm{E}-08)$ & $(6,1 \mathrm{E}-08)$ & $(6,08 \mathrm{E}-08)$ \\
In(Investimento/GDP) & $2,52 \mathrm{E}-05$ & 0,014 & 0,017 \\
& $(0,026)$ & $(0,024)^{*}$ & $(0,025)$ \\
\hline
\end{tabular}

\footnotetext{
${ }^{16}$ Uma hipótese que pode ser feita com respeito aos resultados para volatilidade e depreciação do câmbio para o crescimento do produto nos países emergentes é que eles refletem uma combinação utilizada para estratégias de crescimento Export-led Growth, típica nos países da amostra dos emergentes.
} 


\begin{tabular}{l|r|r|r}
\hline Volatilidade Cambial & $-0,031$ & $-0,032$ & $-0,033$ \\
In(Depreciação Cambial) & $(0,005)^{*}$ & $(0,005)^{*}$ & $(0,005)^{*}$ \\
& 0,106 & 0,101 & 0,107 \\
Regime Fixo & $(0,053)^{*}$ & $(0,054)^{*}$ & $(0,054)^{*}$ \\
Regime Flexível & 0,031 & $\ldots$ & $\ldots$ \\
& $(0,018)^{*}$ & $\ldots$ & $\ldots$ \\
Regime Intermediário & $\ldots$ & $-0,047$ & $\ldots$ \\
& $\ldots$ & $(0,049)$ & $-0,001$ \\
Intercepto & $\ldots$ & $\ldots$ & $(0,020)$ \\
& 0,339 & 0,317 & 0,361 \\
\hline Teste AR(1) & $(0,116)^{*}$ & $(0,125)^{*}$ & $(0,122)^{*}$ \\
Teste AR(2) & 0,000 & 0,000 & 0,000 \\
Teste Hansen & 0,04 & 0,032 & 0,021 \\
Teste Hansen-in-difference & 0,819 & 0,252 & 0,212 \\
\hline N. de observações & 0,001 & 0,677 & 0,734 \\
\hline Font:Resur & 433 & 433 & 433 \\
\hline
\end{tabular}

Fonte: Resultados da pesquisa.Os números entre parênteses são os desvios-padrão e * indica pelo menos $5 \%$ de nível de significância.

OBS.: As variáveis de regimes cambiais e volatilidade cambial são tratadas como endógenas pelos instrumentos defasados e diferenciados no System-GMM.

Para os países desenvolvidos, a variável de volatilidade cambial é igualmente negativa e significante para as especificações i e ii. Para especificação iii a volatilidade não foi estatisticamente significante, o que limita o poder explicativo do trabalho sobre o tema da volatilidade para este conjunto de países.

Chamam atenção ainda outros dois aspectos. Em primeiro lugar, o coeficiente para depreciação cambial - ainda que significante para as três especificações tem valor bastante inferior ao verificado para os países emergentes. Isto sugere que a depreciação cambial seja mais importante para a explicação do crescimento nos emergentes do que nos desenvolvidos, o que em última análise corrobora os elementos destacados no início do trabalho sobre a importância da taxa de câmbio na estratégia de export led growth seguida por boa parte dos países emergentes analisados no trabalho.

Em segundo lugar, chama a atenção o fato de que as variáveis que descrevem regimes cambiais não apareçam estatisticamente significantes para os países desenvolvidos. A princípio, poder-se-ia defender a ideia de que o regime cambial não é importante, em termos de resultados de crescimento, para os países desenvolvidos como o é para os países emergentes. 
Tabela 5: Resultados do Painel Dinâmico de Crescimento para Países Desenvolvidos

\begin{tabular}{|c|c|c|c|}
\hline & (i) & (ii) & (iii) \\
\hline \multirow[t]{2}{*}{ PIB per capita_1 } & 0,937 & 0,778 & 0,727 \\
\hline & $(0,081)^{*}$ & $(0,257)^{*}$ & $(0,031)^{*}$ \\
\hline \multirow[t]{2}{*}{ Crescimento Demográfico } & $6,32 \mathrm{E}-06$ & $2,28 \mathrm{E}-05$ & 8,0E-05 \\
\hline & $(8,24 \mathrm{E}-06)$ & $(2,58 \mathrm{E}-05)$ & $(3,3 \mathrm{E}-05)^{*}$ \\
\hline \multirow[t]{2}{*}{ In(Investimento/GDP) } & 0,070 & 0,127 & 0,210 \\
\hline & $(0.024)^{*}$ & $(0,043)^{*}$ & $(0,063)^{*}$ \\
\hline \multirow[t]{2}{*}{ Volatilidade Cambial } & $-0,091$ & $-0,117$ & $-0,066$ \\
\hline & $(0,054)^{*}$ & $(0,048)^{*}$ & $(0,070)$ \\
\hline \multirow[t]{2}{*}{ In(Depreciação Cambial) } & 0,041 & 0,060 & 0,023 \\
\hline & $(0,044)$ & $(0,047)$ & $(0,048)$ \\
\hline \multirow[t]{2}{*}{ Regime Fixo } & 0,009 & $\ldots$ & $\ldots$ \\
\hline & $(0,008)$ & $\ldots$ & $\ldots$ \\
\hline \multirow[t]{2}{*}{ Regime Flexível } & $\ldots$ & 0,007 & $\ldots$ \\
\hline & $\ldots$ & $(0,050)$ & $\ldots$ \\
\hline \multirow[t]{2}{*}{ Regime Intermediário } & $\ldots$ & $\ldots$ & 0,030 \\
\hline & $\ldots$ & $\cdots$ & $(0,098)$ \\
\hline \multirow[t]{2}{*}{ Intercepto } & 0,276 & 0,161 & 0,268 \\
\hline & $(0,470)$ & $(1,538)$ & $(1,655)^{*}$ \\
\hline Teste AR(1) & 0,006 & 0,005 & 0,006 \\
\hline Teste AR(2) & 0,119 & 0,542 & 0,543 \\
\hline Teste Hansen & 0,997 & 0,997 & 0,997 \\
\hline Teste Hansen-in-difference & 0,995 & 0,996 & 0,996 \\
\hline N. de observações & 340 & 340 & 340 \\
\hline
\end{tabular}

Esse resultado encontra sutil ligação com a ideia de fear of floating. A experiência com regimes cambiais durante episódios de alta descontrolada de preços e a volatilidade cambial associada dos países emergentes podem explicar o uso do controle do câmbio como forma de preservar o produto durante turbulências financeiras, justificando a relação positiva entre câmbio fixo e crescimento do produto para estes. A relação negativa entre crescimento e os regimes cambiais flexíveis, de um lado, e a não significância do regime flexível para o crescimento, por outro lado, sugerem que para os países emergentes a utilização de regimes de câmbio fixo ou gerenciados representaram um fator positivo de crescimento.

O resultado final sugere que os países emergentes podem sofrer de medo de flutuar: a potencial volatilidade cambial resultante de regimes mais flexíveis tem resultados negativos para o produto, fazendo com que esses países tenham motivações para administrar o câmbio de alguma forma, obtendo resultados positivos 
para o crescimento. Além disso, essa potencial rationale estratégica dos países emergentes pode estar associada à prática de promoção de exportações de muitos países emergentes. Para os países desenvolvidos, por outro lado, os regimes cambiais não têm relação significante com crescimento, embora a volatilidade do câmbio seja igualmente um fator negativo para o crescimento.

\section{CONSIDERAÇÕES FINAIS}

Este trabalho teve como objetivo central discutir o papel da taxa de câmbio e de suas flutuações no processo de crescimento econômico. Sua inspiração teórica advém das contribuições de inspiração keynesiana que advogam em prol da importância da variável para o desenvolvimento de um país. De forma geral, pode-se afirmar que os resultados empíricos obtidos do modelo econométrico utilizado ao longo do trabalho corroboram a importância da taxa de câmbio para o crescimento das economias emergentes e desenvolvidas.

Os resultados empíricos obtidos para os países selecionados sugerem que a taxa de câmbio real apresenta-se como uma variável relevante para a compreensão do processo de crescimento econômico. Tanto a volatilidade da taxa de câmbio quanto a depreciação relativa com relação ao equilíbrio de longo prazo foram estatisticamente significantes e apresentaram coeficientes relativamente elevados. Os resultados em relação à escolha dos regimes cambiais, ainda que estaticamente significantes (exceto para os regimes intermediários), foram menos expressivos, tendo em vista o reduzido valor encontrado para os coeficientes.

Para os países emergentes, tanto a depreciação relativa com relação ao equilíbrio de longo prazo quanto a volatilidade da taxa de câmbio real apresentaram-se relevantes para o crescimento econômico. A escolha do regime cambial também se apresentou estatisticamente significativa. No entanto, a título de conclusão deve-se salientar que a depreciação relativa foi a variável mais importante para o crescimento. A volatilidade cambial e a escolha do regime de câmbio, respectivamente, foram menos relevantes. Estes resultados corroboram a discussão teórica estabelecida na primeira parte do trabalho, particularmente a visão de que a depreciação da taxa de câmbio real se constituiu numa peça importante do modelo de crescimento do tipo export led seguido por um amplo conjunto de economias emergentes.

Em relação às economias desenvolvidas, caberia destacar que a depreciação relativa da taxa de câmbio real apresentou-se significativa, porém menos importante do que nas economias emergentes. Isto sugere que o crescimento nestas economias dependeu relativamente menos do nível da taxa de câmbio. Finalmente, as variáveis que descrevem regimes cambiais não apareceram estatisticamente significantes para os países desenvolvidos.

Ainda que não seja o objetivo central deste trabalho, os resultados obtidos, somados à discussão teórica existente, sugerem que o comportamento da taxa de câmbio real deve ser observado com extremo cuidado pelos formuladores de política econômica. As evidências empíricas sugerem que, particularmente para os 
países emergentes, é de fundamental importância a manutenção de uma taxa de câmbio real que mantenha a competitividade externa. Da mesma forma, os resultados sugerem que os formuladores de política econômica devem estar atentos à volatilidade da taxa de câmbio, tendo em vista seus efeitos negativos sobre o crescimento.

Em síntese, os resultados empíricos encontrados neste trabalho sustentam a concepção teórica de inspiração keynesiana que argumenta pela importância da taxa de câmbio real como elemento importante para a trajetória de crescimento, sobretudo nos países emergentes. Por fim, o trabalho também contribui para recuperar um elemento essencial do debate econômico, quer seja, que existem diferenças no processo de crescimento de países emergentes em relação ao dos desenvolvidos.

\section{REFERÊNCIAS BIBLIOGRÁFICAS}

AGHION, P. et al. (2004) Volatility and growth: financial development and the cyclical composition of investment. mimeo.

AGHION, P. BACCHETTA, P. RANCIERI, R. ROGGOF, K. (2006) "Exchange rate volatility and productivity growth: The role of financial development". NBER Working Paper, 12117. Disponível em: http://www.nber.org/papers/w12117. Acesso em: 20 jun. 2007.

ANGELETOS, G. (2003) "Comments on Benigno and Woodford's 'optimal monetary and fiscal policy'”, forthcoming in Mark Gertler and Kenneth Rogoff (eds.), NBER Macroeconomics Annual 2003.

ARELLANO. A., BOND. B. (1991) "Some test of specification for panel data: Monte Carlo evidence and an application to employment equations", Review of Economic Studies, n ${ }^{\circ}$ 58, pp. 277-297.

BALTAGI, B. (2005) Econometric Analisys of Panel Data. Chichester, West Sussex.John Wiley e Sons.

BARRO, R. \& SALA-i-MARTIN, X. (2995) Economic Growth, New York. McGraw-Hill Inc.

BITTENCOURT, M.V.L. (2004) "The impacts of trade liberalization and macroeconomic instability on the Brazilian economy". (Ph.D. Dissertation). The Ohio State University.

BLUNDELL, $\mathrm{R}$ et al. (2000) "Estimation in dynamic panel data models: Improving on the performance of thestandard GMM estimators", The Institute for Fiscal Studies, Working Paper, $\mathrm{n}^{\circ} 12$.

BOND, S. (2001) "GMM estimation of empirical growth models". University of Oxford, Institute for Fiscal Studies, 2001.

BRESSER-PEREIRA, L. C. (2007) Macroeconomia da Estagnação: Crítica da Ortodoxia Convencional no Brasil pós-1994, São Paulo: Editora 34.

BRESSER-PEREIRA, L. C. (2005) “The two methods of economics”. Disponível em: http//www.bresserpereira.org.br. Acesso em: 30 jun. 2007

BRODA, C. ROMALIS, J. (2003) Identifying the Relationship Between Trade and Exchange Rate Volatility. Disponível em: http://faculty.chicagobooth.edu/john.romalis/research/erv_trade.pdf. Acessado em: 30 jun. 2007. CALVO, G. A., REINHART, C. M. (2002) "Fear of floating", The Quarterly Journal of Economics, Vol. CXVII, Issue 2, pp. 379-408.

CERMEÑO, R. e SANIN, M. (2005) "Exchange rate arrangements and volatility of real exchange rate depreciation: panel Evidence for the G7 and 8 Latin American countries", Documento de Trabajo ${ }^{\circ} 297$, CIDE.

CHOI, C. (2000) "Exchange rates, exchange rate volatility and investment in Korea: An empirical investigation", The Bank of Korea Economic Papers. Vol. 4, n ${ }^{\circ} 1$.

DAVIDSON, P. (2002) Financial Markets, Money and the Real World. Northampton, Ma: Edward 
Elgar.COUDERT, V. E COUHARDE, C. (2008), "Currency misalignment and exchange rate regimes in emerging and developing countries”, Working Paper CEPII, $\mathrm{n}^{\mathrm{a}}$ 2008/07.

EDWARDS, S. (2006) "The relationship between exchange rates and inflation targeting revisited" $\mathrm{Na}$ tional Bureau of Economic Research Working Paper, $\mathrm{n}^{\circ} 12163$.

EICHENGREEN, B. (2004) "Global imbalances and the lessons of Bretton Woods", National Bureau of Economic Research (NBER). Working Paper, n 10497.

GHOSH, S.; MELHEM, R.; MOSSE, D. (1997) "Fault-tolerance through scheduling of aperiodic tasks in hard real-time multiprocessor systems". IEEE Trans. Parallel Distrib. Syst., 8(3): 272-284.

GREENSPAN, A. A Era da Turbulência. Elsevier Editora, Rio de Janeiro, 2008.

HO e McCAULEY, R. (2002) "Living with flexible exchange rates: issues and recent experience in inflation targeting emerging markets economy", BIS Working Paper, $\mathrm{n}^{\circ} 130$.

JONES, L.; MANUELLI, R.; STACCHETTI, E. (2000) "Technology and policy shocks in models of endogenous growth", Federal Reserve Bank of Minneapolis Working Paper, $\mathrm{n}^{\circ} 281$.

KEYNES, J. M. (1964) Teoria Geral do Emprego, do Juro e da Moeda. São Paulo: Ed. Atlas.

LEVINE, R.; RENELT, P. (1992) "A sensitivity analysis of cross-country growth regressions", American Economic Review, ${ }^{\circ}$ 82, pp. 942-963.

LEVY-YEYATI, E; STURZENEGGER, F. (2003) "To float or to fix: Evidence on the impact of exchange rate regimes on growth", American Economy Review, $n^{\circ}$ 93, pp. 1173-1193.

NETTO, D. A. (2005) “Meio século de economia brasileira: Desenvolvimento e restrição externa”. In Giambiagi et al., orgs. Economia Brasileira Contemporânea (1945-2004). Rio de Janeiro: Editora Campus.

PALMA, G. (2005) "Four sources of "de-industrialization" and a new concept of Dutch Disease". In: Beyond Reforms: Structural Dynamics and Macroeconomic Vulnerability. Stanford: Stanford University Press/World Bank.

PENEDER, M. (2004) “Tracing empirical trails of Schumpeterian development". Papers on Economics and Evolution, ${ }^{\circ} 409$, pp. 1-20.

PEREE, E.; STEINHERR, A. (1989) "Exchange rate uncertainty and foreign trade”, European Economic Review, n 33, pp. 1241-1264.

RAMEY, G; V. RAMEY (1995) “Cross-country Evidence on the link between volatility and growth”, American Economic Review, n85, pp.1138-1151.

REINHART, C.; ROGOFF, K. (2002) "The modern history of exchange rate arrangements: A reinterpretation", National Bureau of Economic Research Working Paper $n^{\circ} 8963$.

SALA-i-MARTIN, X. (1997) "I just ran two million regressions", American Economic Review, $\mathrm{n}^{\circ} .87$, pp. $178-183$.

SERVÉN, L. (2002) "Real exchange rate uncertainty and private investment in developing countries", World Bank Policy Research Working Paper, $\mathrm{n}^{\circ} 2823$.

TENREYRO, S.; BARRO, R. (2002) “Economic effects of currency unions”, National Bureau of Economic Research Working Paper $n^{\circ} .9435$.

TENREYRO, S. (2003) "On the trade impact of nominal exchange rate volatility", Federal Reserve Bank of Boston Working Papers: 03-2.

THIRLWALL, A. P. (1979) "The balance of payments constraint as an explanation of international growth rate differences”, Banca Nazionale del Lavoro Quarterly Review, pp. 45-53. 